AIAA-2002-1719

\title{
MODELING OF HIGH ASPECT RATIO ACTIVE FLEXIBLE WINGS FOR ROLL CONTROL
}

\author{
Carlos E. S. Cesnik* \\ University of Michigan, Ann Arbor, Michigan \\ and \\ Eric L. Brownt \\ Massachusetts Institute of Technology, Cambridge, Massachusetts
}

\begin{abstract}
The study of wing-warping roll control for high-altitude long-endurance vehicle wings is the primary objective of this paper. For that, a formulation is developed for the preliminary design of high aspect ratio active flexible wings. The formulation captures the nonlinear (large) deflection behavior of the wings, the effects of anisotropic piezoelectric composites embedded in the skin, and the unsteady subsonic aerodynamic forces acting on the wing. Because the wing is long and slender, it can be modeled as a beam undergoing three dimensional displacements and rotations. The cross sectional stiffness, inertia, and actuation properties of the wing are calculated along the span, and then incorporated into the 1-D nonlinear beam model derived in this paper. Finite-state unsteady subsonic airloads are incorporated to complete the state space aeroelastic model. The result is a low order model, capable of representing the important aeroelastic behavior, suitable for preliminary structural design and control synthesis. The capabilities of the formulation are exemplified at the end. Among other things it is shown that the wing-warping control with embedded anisotropic piezocomposite actuators can be more effective than conventional aileron for roll control.
\end{abstract}

\section{Introduction}

In the design of aircraft wings, the goal is usually to minimize the weight of the aircraft structure for a given payload weight. In the optimal design process, the structural material of the wings is reduced until design constraints become active. Reducing the weight of the structure tends to produce wings that are more flexible,

\footnotetext{
* Associate Professor of Aerospace Engineering. Associate Fellow, AIAA. Member, AHS.

${ }^{\mathrm{t}}$ Graduate Research Assistant. Mechanical Engineering.

Copyright $\odot 2002$ by the authors. Published by the American

Institute of Aeronautics and Astronautics, Inc., with permission.
}

making them prone to aeroelastic instability. At the optimal design point, it is possible that one or more of the constraints associated with strength and aeroelastic stability are active. Furthermore, flexible wings will undergo large deflections, making their aeroelastic response difficult to analyze and control using linear methods. Replacing the conventional flaps and ailerons with conformable surfaces with embedded anisotropic piezoelectric materials in the skin may lead to lighter designs. Two main reasons for that are:

1. The actuatuors are integral to the structure, and contribute to its overall stiffness; it eliminates the extra weight associated with hydraulic lines and mechanical linkages.

2. The active wings will be able to extend the flutter and divergence boundaries through active control, allowing for a new optimal design point to be reached, increasing the feasible design space.

Structural design of aircraft wings is an inherently iterative process. With flexible wings, this is complicated by the fact that the lift characteristics depend on the deformed shape of the wing, which cannot be accurately computed without simulation. Knowledge of how the active and passive composite constructions affect the overall aeroelastic behavior is critical for a successful design. In order for the active skin plies to have the mechanical coupling authority for producing the desired deformation, the active plies must contribute to the overall stiffness of the wings. Varying the active composite ply orientations in order to produce higher twist authority may make the wings less stiff in bending and lead to higher static deflections and aeroelastic instability. The design of the active part of the structure should be considered within the overall context of aeroelastic design.

The active wing may be able to exploit aeroelastic tailoring concepts to the limit, using wing flexibility to 
improve performance rather than degrade it. At high dynamic pressure, flaps and ailerons may experience control reversal, due to the aerodynamic twisting moment experienced by the wing when the flaps are deflected. High frequency control surface actuation may excite structural or aeroelastic modes of the complete aircraft, or may produce no effect at all. Active induced-strain actuated wings may have the ability to overcome some of these problems.

There are different studies found in the literature that address some of the issues associated with highlyflexible active wings as discussed in this paper. One of the first active aeroelastic wing studies was conducted by Crawley and co-workers (e.g., Ref. [1]). They examined dominant issues in the preliminary design of a strain actuated aeroelastic wing. Scaling parameters were used to characterize the authority and effectiveness of the piezoelectric actuation. They studied the effects of composite fiber angle and sweep and used isotropic piezoelectric wafers to exploit bendtwist couplings for producing wing twist. The resulting active wing hardware was studied at NASA Langley under the PARTI wing program ${ }^{2}$.

Librescu and co-workers have studied the use of adaptive materials to control the effects of pressure pulse loading on wings ${ }^{3}$. In this study, the wings were modeled as doubly tapered thin walled beams, taking into account the effects of transverse shear, warping inhibition, material anisotropy, and the effects of piezoactuators spread over the surface. The results detail the effects of wing parameters, such as ply angles, tapering, and actuator size, on the response due to various excitations.

Khot and co-workers have explored the active aeroelastic wing concept ${ }^{4}$ by using elastic twist and camber deformation in a fighter-type wing (e.g., Ref. [5]) The goal was to enhance roll maneuver performance at high dynamic pressures. Therein, a fullscale conventional wing construction was considered for the assessment of the strain energy required to produce the antisymmetric twist and camber deformation needed for a given roll performance. The actuators that would generate the deformation were not defined in the study and therefore their effects on the aeroelastic characteristics of the wing could not be accounted.

Jones and co-workers have worked on high-altitude long-endurance (HALE) vehicle design during the last decade (e.g., Ref. [6]). They describe some of the design challenges associated with these vehicles. Among other things, they show that standard initial aircraft design techniques such as EDSU data sheets are not applicable for these wings as the altitude and aspect ratio increase.

Patil and co-workers have studied the nonlinear behavior of flexible wings undergoing large deflections. In Ref. [7], the necessity for including higher-order nonlinear effects to accurately model the aeroelastic behavior is detailed. In particular, a linear aeroelastic analysis based on the undeformed geometry will lead to erroneous results when the wings are highly flexible. To accurately model the behavior, the wings must be brought to their nonlinear equilibrium condition before the analysis can proceed. From there, a stability analysis can be performed on the linearized model. It was found that the flutter speed tends to decrease when the wing tip displacements increase. This effect can only be obtained if the wings are first brought to the nonlinear static equilibrium through an iterative process. The main reasons for the changes in aeroelastic behavior as the wings deform are that both the structural modes and the influence of aerodynamic forces onto the structure change considerably as the deformation increases. The first chord-wise bending mode of the straight wing, for instance, will tend towards a ì rockingî mode as the tip deflection increases, and may coalesce with the first torsion mode. Another important result of Ref. [7] is that the total lift predicted from a linear model will generally be higher than the actual lift. As the wing deforms, the direction of the lift forces rotate with the wing, and do not remain parallel.

In Ref. [8], Drela modeled the complete flexible aircraft as an assemblage of joined nonlinear beams and applied aerodynamic strip theory. The resulting nonlinear equation set is solved by a full Newton method. Through simplifications of the model, the computational size is made small enough for interactive preliminary design.

Cesnik and Ortega-Morales present a framework for studying the effects of combined bending and twisting actuation on the aeroelastic performance of highlyflexible active composite wings ${ }^{9,10}$. Therein, the nonlinear active aeroelastic analysis consists of an asymptotically correct active cross section formulation, geometrically-exact mixed formulation for dynamics of moving beams, and a finite-state unsteady aerodynamics model. LQG controllers were designed to alleviate gust loads and to extend the flutter boundary.

To navigate the rich structural design space of active flexible wings requires a large degree of iteration. The ongoing work intends to develop a framework for the integrated active structure and control design for 
studying the behavior of these wings. The derivation of the equations of motion for the two-wing active roll model is discussed in this paper. First a nonlinear beam model is derived in which the effects of embedded anisotropic piezoelectric strain actuation are taken into account. Then the principle of virtual work is employed to arrive at the equations of motion for the rigid roll/flexible wing structure. A finite-state unsteady airloads model ${ }^{11,12}$ is incorporated to complete the aeroelastic model. Finally, the equations are developed in state-space form to facilitate control synthesis.

\section{Formulation}

For the present study, the vehicle is treated as a rigid body free to roll. The wings are allowed fully coupled three-dimensional bending and twisting deformation. It is assumed that extensional and shear deformations play a negligible role in the overall wing deformation.

\section{$\underline{\text { Kinematics }}$}

Three reference frames are used in this study. The global frame is represented by $a$, with gravity pointing in the negative $z$ direction. The body frame, $b$, is fixed to the fuselage, and is initially aligned with frame $a$ before being taken through two rotations. First, as shown in Fig. 1, the body is rotated about the $x$ axis by $\alpha_{b}$, the body angle of attack. Then the body is rotated about its $y$-axis by $\phi$, the roll angle. The orientation of the body with respect to the global frame is given by

$$
C^{a b}=\left[\begin{array}{ccc}
1 & 0 & 0 \\
0 & \cos \alpha_{b} & -\sin \alpha_{b} \\
0 & \sin \alpha_{b} & \cos \alpha_{b}
\end{array}\right]\left[\begin{array}{ccc}
\cos \phi & 0 & \sin \phi \\
0 & 1 & 0 \\
-\sin \phi & 0 & \cos \phi
\end{array}\right]
$$

Angle $\alpha_{b}$ is fixed and is not considered a motion variable in this study.

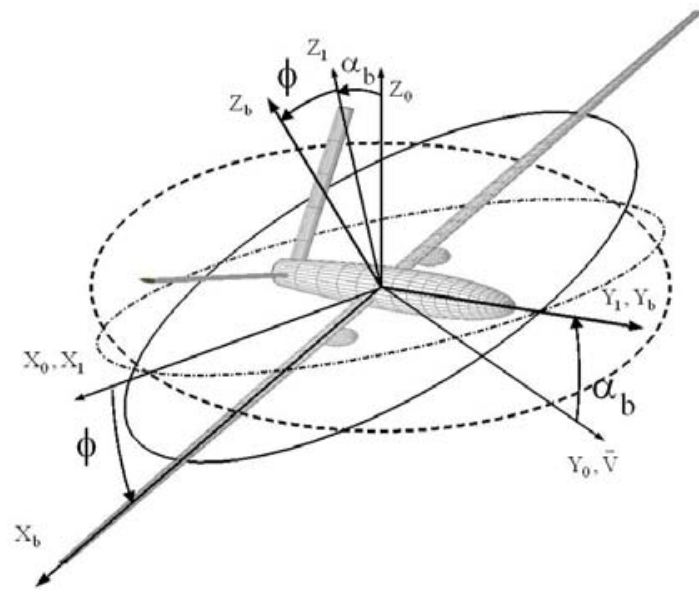

Figure 1: Global and Body Coordinate Systems
The local beam (wing) coordinate system, $w$, is constructed from the local unit direction vectors, $u_{x}, u_{y}$, and $u_{z}$, attached to the deformed beam reference line as shown in Fig. 2. The direction vectors are defined with respect to frame $b$ unless otherwise subscripted.

The vehicle velocity with respect to the body frame is given by

$$
V_{b}=C^{b a} V_{a}
$$

where $V_{a}$ is the velocity in the global frame.

Since the wings are long and slender, they can be well modeled as beams undergoing fully coupled threedimensional deformation. Because of the large deformation due to wing flexibility, a nonlinear formulation is derived that describes the motion of the reference line. The beam reference line is treated as a three dimensional line of constant length, with bending and torsion deformation. The shape of the line is determined by the distribution of curvature, $\kappa_{x}(s), \kappa_{y}(s), \kappa_{z}(s)$.

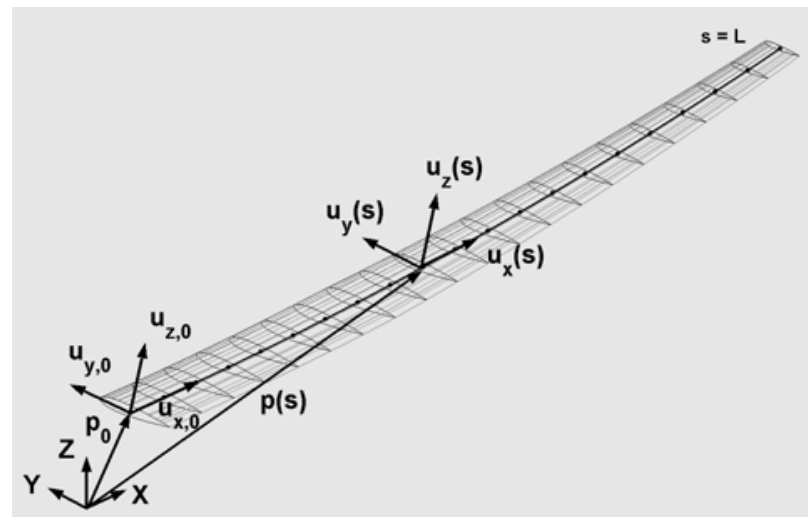

Figure 2: Wing Coordinate System

Assuming that the wing deformation is dominated by bending and twisting deformation, i.e., the extensional and shear strains play a negligible role in the gross deformation, the beam direction vectors can be obtained from the differential equations

$$
\begin{aligned}
& \frac{d}{d s} u_{x}(s)=\kappa_{z}(s) u_{y}(s)-\kappa_{y}(s) u_{z}(s) \\
& \frac{d}{d s} u_{y}(s)=\kappa_{x}(s) u_{z}(s)-\kappa_{z}(s) u_{x}(s) \\
& \frac{d}{d s} u_{z}(s)=\kappa_{y}(s) u_{x}(s)-\kappa_{x}(s) u_{y}(s)
\end{aligned}
$$

The above equation is expressed in a more compact form as

$$
\frac{d}{d s} u(s)=K(s) u(s)
$$




$$
u=\left\{\begin{array}{l}
u_{x} \\
u_{y} \\
u_{z}
\end{array}\right\}, K=\left[\begin{array}{c|c|c}
0 & I \kappa_{z} & -I \kappa_{y} \\
\hline-I \kappa_{z} & 0 & I \kappa_{x} \\
\hline I \kappa_{y} & -I \kappa_{x} & 0
\end{array}\right]
$$

and $I$ is the $3 \times 3$ identity matrix. The transformation matrix from the local deformed wing frame to the body frame is constructed from the local direction vectors

$$
C^{w b}(s)=\left[\begin{array}{ccc} 
& & \vdots \\
u_{x}(s) & u_{y}(s) & u_{z}(s) \\
: & : & :
\end{array}\right]
$$

A vector expressed in frame $w$, can be expressed in frame $b$ by the transformation

$$
v_{b}=C^{w b} v_{w}=v_{w_{x}} u_{x}+v_{w_{y}} u_{y}+v_{w_{z}} u_{z}
$$

The position, $p(s)$,of a point on the reference line can be found from the relation

$$
\frac{d}{d s} p(s)=u_{x}(s)
$$

Given the initial condition at the wing root, the shape of the wing is completely defined by the distribution of curvature along the beam coordinate, $s$.

Using curvature as the primary kinematic variables has several advantages. First, the shape of the reference line is completely defined by three variables along the coordinate, $s$, instead of six to twelve required when displacements and rotations are used as the independent variables. Since no extra variables are used to define the shape of the reference line, the need for constraint equations is eliminated. Second, when solving for the motion of the reference line, no singularities exist in the solution. Furthermore, it is generally more difficult to solve for the strain variables from the displacement variables than vice-versa.

\section{Virtual Work Formulation}

The total virtual work done on the vehicle is found by integrating over the volume all internal and external forces times their corresponding displacements, and is given in a general form by

$$
\delta W=\int_{V}(f(x, y, z) \delta \zeta(x, y, z)) d V
$$

where $f$ represents all generalized forces acting on a differential volume element, including the inertial forces distributed over the element, pressure forces applied to any free surfaces, and internal elastic and piezoelectric stresses, and $\delta \zeta$ is the corresponding virtual displacement.

This equation can be simplified through the assumed kinematical and constitutive relations of the present problem. The fuselage is treated as a rigid body with only roll degree of freedom and center of gravity on the roll axis, and the wings as beams (lines with mass, inertia, and stiffness) with three dimensional curvature, so the virtual work done on the vehicle is written as

$$
\begin{aligned}
& \delta W=-\delta \phi I_{f u s} \ddot{\phi} \\
& +\left.\int_{s=0}^{L}\left(\delta p^{T} F+\delta \theta^{T} M+\delta \kappa^{T} M^{\text {int }}\right) d s\right|_{\text {right wing }} \\
& +\left.\int_{s=0}^{L}\left(\delta p^{T} F+\delta \theta^{T} M+\delta \kappa^{T} M^{\text {int }}\right) d s\right|_{\text {left wing }}
\end{aligned}
$$

The kinematic variables that describe the rigid and flexible motion of the vehicle are

$$
q=\left\{\kappa\left(s_{R}\right), \kappa\left(s_{L}\right), \phi\right\}
$$

where thte subscripts $R$ and $L$ correspond to quantities associated with the right and left wing, respectively. Furthermore, it is assumed that the curvature variables can be interpolated from discrete values at linearly spaced coordinates along the beam reference line. So that the complete set of discrete kinematic variables is given by

$$
\underline{q}=\left\{\begin{array}{c}
\underline{\kappa}_{R} \\
\underline{\kappa}_{L} \\
\phi
\end{array}\right\}
$$

where $\underline{\kappa}_{R}$ and $\underline{\kappa}_{L}$ are columns containing the $\{x, y, z\}$ curvature variables at the node locations, and $\phi$ is the roll angle. For the rest of this paper, the notation (@) will be used to denote a column vector of nodal quantities.

The position and orientation of the local coordinate system fixed to the beam reference line are dependent variables given by

$$
\begin{array}{ll}
\underline{P}_{R}=\underline{P}\left(\underline{\kappa}_{R}\right) & \underline{P}_{L}=\underline{P}\left(\underline{\kappa}_{L}\right) \\
\underline{u}_{x, R}=\underline{u}_{x, R}\left(\underline{\boldsymbol{\kappa}}_{R}\right) & \underline{u}_{x, L}=\underline{u}_{x, L}\left(\underline{\boldsymbol{\kappa}}_{L}\right) \\
\underline{u}_{y, R}=\underline{u}_{y, R}\left(\underline{\boldsymbol{\kappa}}_{R}\right) & \underline{u}_{y, L}=\underline{u}_{y, L}\left(\underline{\boldsymbol{\kappa}}_{L}\right) \\
\underline{u}_{z, R}=\underline{u}_{z, R}\left(\underline{\boldsymbol{\kappa}}_{R}\right) & \underline{u}_{z, L}=\underline{u}_{z, L}\left(\underline{\boldsymbol{\kappa}}_{L}\right)
\end{array}
$$

where $P_{i}$ is the position of the $i t h$ node with respect to the body coordinate system, fixed at the fuselage centerline and $u_{x, i}, u_{y, i}$, and $u_{z, i}$ are the orthogonal unit 
direction vectors that define the local coordinate system at the $i t h$ node.

The specific terms in the integration of Eq. (10) will be described later and the result is given by

$$
\delta W=\delta \underline{q}^{T}\left(-M_{q} \underline{\ddot{q}}-C_{q} \underline{\dot{q}}-K_{q} \underline{q}+\underline{R}_{q}\right)
$$

where $M_{q}, C_{q}$, and $K_{q}$ are the mass, damping, and stiffness matrices, respectively, and $\underline{R}_{q}$ contains the effects of external loads and applied voltage to the piezoelectric plies within the wing construction. In Eq. (14), the total virtual work contributed by each virtual displacement must be zero, since the virtual displacements are arbitrary. Then, the term in parentheses must vanish, giving the second order structure equations of motion

$$
M_{q} \underline{\ddot{q}}+C_{q} \underline{\dot{q}}+K_{q} \underline{q}=\underline{R}_{q}
$$

The system matrices and load vector are described next.

\section{Beam Discretization}

The reference axis is discretized at $n_{k}$ equally spaced coordinates, $\underline{s}_{k}$, between which the curvature is assumed to vary linearly (Fig. 3 ). The $3 n_{k} x l$ column matrix of nodal curvatures, given by

$$
\underline{\kappa}=\left\{\begin{array}{l}
\underline{\kappa}_{x} \\
\underline{\kappa}_{y} \\
\underline{\kappa}_{z}
\end{array}\right\}
$$

which are the curvature variables that define the beam geometry with respect to the body.

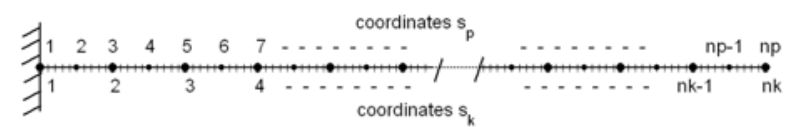

Figure 3: Discretization of the beam reference line

Mid-nodes are used to represent displacement variables so that a second set of coordinates, $\underline{s}_{p}$, will be used frequently. For the purpose of numerical integration, a fine spacing coordinate set, $s_{f}$, is also used. An array of distributed beam properties, $\underline{m}\left(\underline{S}_{p}\right)$, defined at $\underline{s}_{p}$, can be interpolated onto the fine spacing using an interpolating matrix

$$
\underline{m}_{f}=\left[a_{p}^{f}\right] \underline{m}_{p}
$$

The integral of the product of two or more functions of the beam coordinate may be performed using the following relation

$$
\int_{s=0}^{L} h(s) g(s) d s \cong \underline{h}^{T}\left[a_{p}^{f}\right]^{T}\left[a_{p}^{f}\right] \underline{g} \Delta s_{f}
$$

where $\Delta s_{f}$ is the fine coordinate spacing. Similarly, a column matrix of nodal $x, y$, and $z$ vector components is denoted

$$
\underline{F}=\left\{\begin{array}{l}
\underline{F}_{x} \\
\underline{F}_{y} \\
\underline{F}_{z}
\end{array}\right\}
$$

and may be integrated using the matrix relation

$$
\int_{s=0}^{L} F(s) G(s) d s \cong \underline{F}^{T}\left[A_{p}^{f}\right]^{T}\left[A_{p}^{f}\right] \underline{G} \Delta s_{f}
$$

where

$$
\left[A_{p}^{f}\right]=\left[\begin{array}{c|c|c}
a_{p}^{f} & 0 & 0 \\
\hline 0 & a_{p}^{f} & 0 \\
\hline 0 & 0 & a_{p}^{f}
\end{array}\right]
$$

Starting with the position and orientation of the beam root attachment point, and using the assumption that curvature varies linearly between the nodes, an approximate solution to Eq. (4) is obtained by marching the solution forward from the wing root,

$$
\begin{gathered}
u_{i+1}=e^{G_{i}} u_{i} \\
p_{i+1}=p_{i}+\frac{1}{2} \Delta s\left(u_{x, i}+u_{x, i+1}\right)
\end{gathered}
$$

where

$$
G_{i}=\frac{1}{2} \Delta s\left(K_{i}+K_{i+1}\right)
$$

Nodal velocities, accelerations, and partial derivatives can be obtained by differentiation of Eq. (22)

$$
\begin{gathered}
\dot{u}_{i+1}=\dot{G}_{i} e^{G_{i}} u_{i}+e^{G_{i}} \dot{u}_{i} \\
\dot{p}_{i+1}=\dot{p}_{i}+\frac{1}{2} \Delta s\left(\dot{u}_{x, i}+\dot{u}_{x, i+1}\right) \\
\ddot{u}_{i+1}=\ddot{G}_{i} e^{G_{i}} u_{i}+\dot{G}_{i} \dot{u}_{i+1}+e^{G_{i}} \ddot{u}_{i} \\
\ddot{p}_{i+1}=\ddot{p}_{i}+\frac{1}{2} \Delta s\left(\ddot{u}_{x, i}+\ddot{u}_{x, i+1}\right) \\
\frac{\partial u_{i+1}}{\partial \kappa_{x, j}}=\frac{\partial G_{i}}{\partial \kappa_{x, j}} e^{G_{i}} u_{i}+e^{G_{i}} \frac{\partial u_{i}}{\partial \kappa_{x, j}}
\end{gathered}
$$

and the velocities at the root are given by

$$
\begin{gathered}
\dot{u}_{s=0}=\omega_{f} \times u_{s=0} \\
\dot{p}_{s=0}=V+\omega_{f} \times p_{s=0}
\end{gathered}
$$


where $\omega_{f}$ is the fuselage angular velocity. The angular velocity at a node, and the derivative of the $i t h$ rotation variable with respect to the $j$ th curvature variable can be expressed in frame $b$ using the motion of the direction vectors

$$
\dot{\theta}=C^{w b}\left\{\begin{array}{l}
\dot{u}_{y} \cdot u_{z} \\
\dot{u}_{z} \cdot u_{x} \\
\dot{u}_{x} \cdot u_{y}
\end{array}\right\} \quad, \frac{\partial \theta_{i}}{\partial \kappa_{j}}=C_{i}^{w b}\left\{\begin{array}{l}
\frac{\partial u_{y, i}}{\partial \kappa_{j}} \cdot u_{z, i} \\
\frac{\partial u_{z, i}}{\partial \kappa_{j}} \cdot u_{x, i} \\
\frac{\partial u_{x, i}}{\partial \kappa_{j}} \cdot u_{y, i}
\end{array}\right\}
$$

where $\kappa_{j}$ is a nodal curvature variable, as in Eq. (16). The nodal displacement and rotation variables for a wing are related to the curvature and roll variables through a set of kinematic relations,

$$
\begin{aligned}
& \left\{\begin{array}{l}
\delta \underline{P} \\
\delta \underline{u}_{i}
\end{array}\right\}=\left[\begin{array}{c|c}
J_{\kappa}^{p} & J_{\phi}^{p} \\
\hline J_{\kappa}^{u_{i}} & J_{\phi}^{u_{i}}
\end{array}\right]\left\{\begin{array}{c}
\delta \underline{\kappa} \\
\delta \phi
\end{array}\right\} \\
& \left\{\begin{array}{c}
\dot{P} \\
\underline{\dot{u}}_{i}
\end{array}\right\}=\left[\begin{array}{c|c}
J_{\kappa}^{p} & J_{\phi}^{p} \\
\hline J_{\kappa}^{u_{i}} & J_{\phi}^{u_{i}}
\end{array}\right]\left\{\begin{array}{c}
\dot{\kappa} \\
\dot{\dot{\phi}}
\end{array}\right\} \\
& \left\{\begin{array}{c}
\ddot{P} \\
\hdashline \ddot{\underline{u}}_{i}
\end{array}\right\}=\left[\begin{array}{c|c}
J_{\kappa}^{p} & J_{\phi}^{p} \\
\hline J_{\kappa}^{u_{i}} & J_{\phi}^{u_{i}}
\end{array}\right]\left\{\begin{array}{l}
\ddot{\kappa} \\
\ddot{\ddot{\phi}}
\end{array}\right\}+\left[\begin{array}{c|c}
\dot{J}_{\kappa}^{p} & \dot{J}_{\phi}^{p} \\
\hline \dot{J}_{\kappa}^{u_{i}} & \dot{J}_{\phi}^{u_{i}}
\end{array}\right]\left\{\begin{array}{c}
\dot{\kappa} \\
\dot{\phi}
\end{array}\right\}
\end{aligned}
$$

where $i=x, y, z$ and the elements of the Jacobian matrices in these equations are constructed by applying the relationships in Eqs. (22)-(26). The nodal angular velocity and accelerations are determined by applying the relations in Eq. (28).

Coriolis and centrifugal accelerations are accounted for in the velocity terms. Note that in these relations the nodal position and direction vectors, $\underline{P}$ and $\underline{u}$, do not depend on the orientation of the body since they are defined with respect to the body frame. The virtual displacements, velocities, and accelerations, however, are absolute quantities represented in the body frame, and therefore depend on body motion.

\section{Inertia and Gravity Loads}

The inertial and gravitational forces acting on each volume element of the wings and wing stores are considered. The total virtual work is derived in a form which is compatible with the beam motion variables. The position and acceleration of a point, $a$, in a body $A$ can be given by

$$
\begin{aligned}
p_{a} & =p_{A}+x u_{x}+y u_{y}+z u_{z} \\
& =R_{a} h_{A}
\end{aligned}
$$

$$
\begin{aligned}
& R_{a}=\left[\begin{array}{llll}
1 & x & y & z
\end{array}\right] \\
& h_{A}^{T}=\left[\begin{array}{llll}
p_{A}^{T} & u_{x}^{T} & u_{y}^{T} & u_{z}^{T}
\end{array}\right]
\end{aligned}
$$

where $p_{A}$ is the position of the origin of a coordinate system fixed in $A$ with respect to an inertial frame, and $\{x, y, z\}$ is the position of point $a$ in the body, with respect to that local coordinate system.

The inertial and gravitational forces acting on a differential volume element are given by

$$
F_{a}=\rho\left(-\ddot{p}_{a}+g\right) d V=\rho\left(-R_{a} \ddot{h}_{A}+g\right) d V
$$

where $\rho$ is the density and $g$ is the gravity vector. The virtual work done on a volume element in the body due to the inertial and gravitational forces acting on it is given by

$$
\delta W_{a}=\delta p_{a}^{T} F_{a}=\delta h_{A}^{T} \rho\left(R_{a}^{T} g-R_{a}^{T} R_{a} \ddot{h}_{A}\right) d V
$$

The total virtual work acting on body $A$ is found by integrating Eq. (36) over the volume,

$$
\delta W_{A}=\delta h_{A}^{T}\left(N_{A} g_{A}-M_{A} \ddot{h}_{A}\right)
$$

where

$$
N_{A}=\left[\begin{array}{c}
m \\
\hdashline m r_{x} \\
\hdashline m r_{y} \\
\hdashline m r_{z}
\end{array}\right], M_{A}=\left[\begin{array}{c|c:c:c}
m & m r_{x} & m r_{y} & m r_{z} \\
\hline m r_{x} & A_{x x} & A_{x y} & A_{x z} \\
\hdashline m r_{y} & A_{y x} & A_{y y} & A_{y z} \\
\hdashline m r_{z} & A_{z x} & A_{z y} & A_{z z}
\end{array}\right]
$$

$m$ is the mass of the body, or mass per length for a cross section, $\left\{r_{x}, r_{y}, r_{z}\right\}$ are the center of gravity offsets in the local coordinate system, and the constants $A_{i j}$ are closely related to the elements of the rigid body inertia matrix, $I_{i j}$,

$$
\begin{gathered}
A_{x x}=\int_{V} \rho x^{2} d V=m r_{x}^{2}+\frac{1}{2}\left(I_{y y}+I_{z z}-I_{x x}\right) \\
A_{y y}=\int_{V} \rho y^{2} d V=m r_{y}^{2}+\frac{1}{2}\left(I_{x x}+I_{z z}-I_{y y}\right) \\
A_{z z}=\int_{V} \rho z^{2} d V=m r_{z}^{2}+\frac{1}{2}\left(I_{x x}+I_{y y}-I_{z z}\right) \\
A_{x y}=A_{y x}=\int_{V} \rho x y d V=m r_{x} r_{y}+I_{x y} \\
A_{x z}=A_{z x}=\int_{V}^{V} \rho x z d V=m r_{x} r_{z}+I_{x z} \\
A_{y z}=A_{z y}=\int_{V} \rho y z d V=m r_{y} r_{z}+I_{y z}
\end{gathered}
$$


where $I_{i j}$ are taken about the center of gravity. Eq. (37) represents the total inertial and gravitational virtual work acting on a rigid body, such as an engine, or the virtual work per length acting on a wing cross section. The total virtual work done on the flexible part of the wing structure is found by integrating along the beam reference line, yielding

$$
\delta W=\int_{s=0}^{L} \delta W_{A}(s) d s=\delta \underline{h}^{T}\left(N_{h} \underline{g}-M_{h} \underline{\ddot{h}}\right)
$$

where

$$
M_{h}=\left[\begin{array}{llll}
M_{11} & M_{12} & M_{13} & M_{14} \\
M_{21} & M_{22} & M_{23} & M_{24} \\
M_{31} & M_{32} & M_{33} & M_{34} \\
M_{41} & M_{42} & M_{43} & M_{44}
\end{array}\right] \quad N_{h}=\left[\begin{array}{l}
M_{11} \\
M_{21} \\
M_{31} \\
M_{41}
\end{array}\right]
$$

and $\underline{h}$ is a column matrix of nodal displacement variables. The $M_{i j}$ contain mass properties and performs the integration of Eq. (40), i.e,

$$
\begin{aligned}
& \delta \underline{P}^{T} M_{11} \underline{\ddot{P}}=\int_{s=0}^{L} \delta p(s)^{T} m(s) \ddot{p}(s) d s \\
& \delta \underline{u}_{x}^{T} M_{23} \underline{\ddot{u}}_{y}=\int_{s=0}^{L} \delta u_{x}(s)^{T} A_{x y}(s) \ddot{u}_{y}(s) d s
\end{aligned}
$$

and so on, and are constructed using the relations given by Eqs. (17)-( 21).

Considering all of the vehicle motion, the total virtual work due to inertial forces acting on the vehicle is established

$$
\delta W^{a c c}=\delta \underline{H}^{T}\left(N_{H} \underline{g}-M_{H} \underline{\ddot{H}}\right)
$$

where

$$
\begin{gathered}
\underline{H}^{T}=\left\{\underline{h}_{R}^{T}, \underline{h}_{L}^{T}, \phi\right\} \\
M_{H}=\left[\begin{array}{c|c|c}
M_{h} & 0 & 0 \\
\hline 0 & M_{h} & 0 \\
\hline 0 & 0 & I_{f}
\end{array}\right] \quad N_{H}=\left[\begin{array}{c}
N_{h} \\
\hline N_{h}
\end{array}\right]
\end{gathered}
$$

and $I_{f}$ is the fuselage roll inertia. Eq. (42) can be written in terms of the curvature and roll variables by applying Eqs. (29) and (31), and constructing the Jacobian matrix,

with

$$
\delta \underline{H}=J \delta \underline{q} \quad, \quad \underline{\ddot{H}}=J \underline{\ddot{q}}+J \underline{\dot{q}}
$$

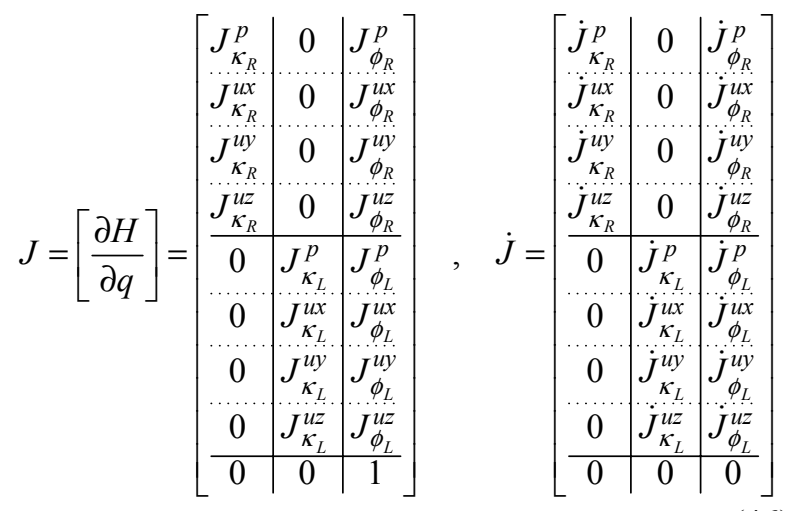

The total virtual work becomes

$$
\delta W^{a c c}=\delta \underline{q}^{T} J^{T}\left(-M_{H} J \underline{\ddot{q}}-M_{H} \dot{j} \underline{\dot{q}}+N_{H} \underline{g}\right)
$$

In this equation, the coriolis and centrifugal effects are contained in the second term in parentheses.

\section{Aerodynamic Loads}

The aerodynamic loads used in the present work are based on the formulation of Refs. [11] and [12]. The theory calculates loads on a thin deformable airfoil undergoing large motion in a subsonic flow, with small deformations about that motion. In the present approach, the airloads are written in terms of the total instantaneous motion of the airfoil, and then a Taylor expansion is used. The lift, moment, and drag are given by

$$
\begin{gathered}
L=2 \pi \rho b\left(\dot{y}\left[\left(\frac{1}{2} b-d\right) \dot{\alpha}-\dot{z}-\lambda_{0}\right]-\frac{1}{2} b \ddot{z}-\frac{1}{2} b d \ddot{\alpha}\right) \\
\begin{array}{c}
M=2 \pi \rho b\left(\dot{y}\left[-\left(d+\frac{1}{2} b\right) \dot{z}-\left(d+\frac{1}{2} b\right) \lambda_{0}-d^{2} \dot{\alpha}\right]\right. \\
\left.-\frac{1}{2} b d \ddot{z}-\frac{1}{2} b\left(d^{2}+\frac{1}{8} b^{2}\right) \ddot{\alpha}\right)
\end{array} \\
D=-2 \pi \rho b\left(\dot{z}^{2}+d^{2} \dot{\alpha}^{2}+\lambda_{0}^{2}+2 d \dot{\alpha} \dot{z}+2 \lambda_{0} \dot{z}+2 d \dot{\alpha} \lambda_{0}\right)
\end{gathered}
$$

where $b$ is the semichord, $d$ is the distance of the midchord in front of the reference axis, and $\lambda_{0}$ is the induced flow due to free vorticity. The motion variables are defined in Fig. 4.

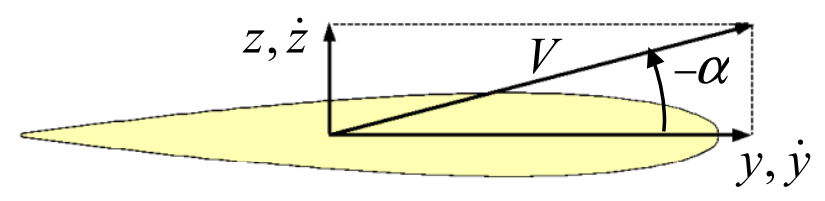

Figure 4: Airfoil motion definition 
The inflow is obtained through the finite-state inflow theory $^{11}$, and is represented in terms of $N$ states $\left(\lambda_{l}\right.$, $\left.\lambda_{2}, \ldots \lambda_{N},\right)$ as

$$
\lambda_{0}=\frac{1}{2} \sum_{n=1}^{N} b_{n} \lambda_{n}
$$

where the $b_{n}$ are found by least squares method, and the $\lambda_{n}$ can be expressed as a set of differential equations, expressed here as

$$
\dot{\lambda}=E_{1} \lambda+E_{2} \ddot{z}+E_{3} \ddot{\alpha}+E_{4} \dot{\alpha}
$$

where the matrices, $E_{i}$, are based on the description given in Ref. [12]. The airloads are linearized about time $t_{a}$ by making the substitutions,

$$
\begin{aligned}
& \dot{y}=\left(\dot{y}_{a}+\Delta \dot{y}\right) \\
& \dot{z}=\left(\dot{z}_{a}+\Delta \dot{z}-\dot{y}_{a} \Delta \alpha\right) \\
& \dot{\alpha}=\left(\dot{\alpha}_{a}+\Delta \dot{\alpha}\right) \\
& \lambda_{0}=\left(\lambda_{0, a}+\Delta \lambda_{0}\right)
\end{aligned}
$$

which to first order gives the linearized lift, moment, and drag as

$$
\begin{aligned}
L & =L_{a}+L_{\dot{\alpha}} \Delta \dot{\alpha}+L_{\dot{y}} \Delta \dot{y}+L_{\dot{z}} \Delta \dot{z} \\
& +L_{\alpha} \Delta \alpha+L_{\lambda_{0}} \Delta \lambda_{0}+L_{\ddot{z}} \ddot{z}+L_{\ddot{\alpha}} \ddot{\alpha} \\
M & =M_{a}+M_{\dot{\alpha}} \Delta \dot{\alpha}+M_{\dot{y}} \Delta \dot{y}+M_{\dot{z}} \Delta \dot{z} \\
& +M_{\alpha} \Delta \alpha+M_{\lambda_{0}} \Delta \lambda_{0}+M_{\ddot{z}} \ddot{z}+M_{\ddot{\alpha}} \ddot{\alpha} \\
D & =D_{a}+D_{\dot{\alpha}} \Delta \dot{\alpha}+D_{\dot{y}} \Delta \dot{y}+D_{\dot{z}} \Delta \dot{z} \\
& +D_{\alpha} \Delta \alpha+D_{\lambda_{0}} \Delta \lambda_{0}+D_{\ddot{z}} \ddot{z}+D_{\ddot{\alpha}} \ddot{\alpha}
\end{aligned}
$$

where the lift terms are given here as

$$
\begin{aligned}
& L_{a}=2 \pi \rho b\left(\left(\frac{1}{2} b-d\right) \dot{y}_{a} \dot{\alpha}_{a}-\dot{y}_{a} \dot{z}_{a}-\dot{y}_{a} \lambda_{0, a}\right) \\
& L_{\dot{\alpha}}=2 \pi \rho b\left(\frac{1}{2} b-d\right) \dot{y}_{a} \\
& L_{\dot{y}}=2 \pi \rho b\left[\left(\frac{1}{2} b-d\right) \dot{\alpha}_{a}-\dot{z}_{a}\right] \\
& L_{\dot{z}}=-2 \pi \rho b \dot{y}_{a} \\
& L_{\alpha}=2 \pi \rho b \dot{y}_{a}^{2} \\
& L_{\lambda_{0}}=-2 \pi \rho b \dot{y}_{a} \\
& L_{\ddot{z}}=-\pi \rho b^{2} \\
& L_{\ddot{\alpha}}=-\pi \rho b^{2} d
\end{aligned}
$$

and similarly for the drag and moment terms.
The virtual work done by these forces acting over the wing is given by

$$
\delta W=\int_{s=0}^{L}(L \delta z+D \delta y+M \delta \alpha) d s
$$

The integration is performed by evaluating the aerodynamic forces and moments at the nodes, and using the interpolation functions so that Eq. (54) can be expressed as

$$
\delta W^{\text {aero }}=\delta \underline{P}^{T} B_{F} \underline{F}^{\text {aero }}+\delta \underline{\theta}^{T} B_{M} \underline{M}^{\text {aero }}
$$

where

$$
\underline{F}^{\text {aero }}=\left\{\begin{array}{c}
\underline{0} \\
\underline{D} \\
\underline{L}
\end{array}\right\} \quad, \quad \underline{M}^{\text {aero }}=\left\{\begin{array}{c}
\underline{M}_{x} \\
\underline{0} \\
\underline{0}
\end{array}\right\}
$$

After some manipulation, the virtual work can be written in terms of the vehicle degrees of freedom as

$$
\delta W^{\text {aero }}=\delta \underline{q}^{T}\left(B_{2}\left\{\begin{array}{l}
\underline{F}_{R}^{\text {aero }} \\
\underline{F}_{L}^{\text {aero }}
\end{array}\right\}+B_{3}\left\{\begin{array}{l}
\underline{M}_{R}^{\text {aero }} \\
\underline{M}_{L}^{\text {aero }}
\end{array}\right\}\right)
$$

where the nodal aerodynamic forces and moments can be linearized about the instantaneous state, $\left\{\underline{q}_{0}, \underline{\dot{q}}_{0}\right\}^{T}$, as

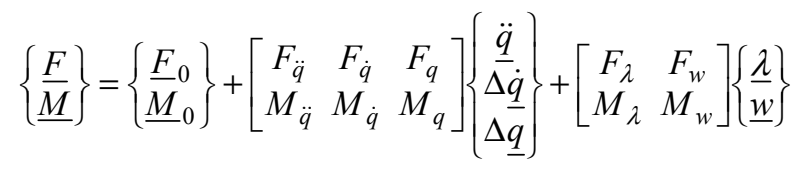

where $\Delta \underline{q}=\underline{q}-\underline{q}_{0}$, and $\underline{q}_{0}$ is the system state where the instantaneous loads are evaluated. Here, gust input, $w$, was introduced, which enters the equations in the same way as the airfoil velocity terms.

Internal Elastic and Piezoelectric Forces and Moments The internal elastic and piezoelectric forces and moments constitutive relation may be obtained from a thin-walled model, such as given in Ref. [13], or from a more general finite element program such as VABS$\mathrm{A}^{14}$, and are symbolic represented by

$$
\left\{\begin{array}{l}
F_{1} \\
M_{1} \\
M_{2} \\
M_{3}
\end{array}\right\}=\left\{\begin{array}{l}
F_{1} \\
M_{1} \\
M_{2} \\
M_{3}
\end{array}\right\}^{e}-\left\{\begin{array}{l}
F_{1} \\
M_{1} \\
M_{2} \\
M_{3}
\end{array}\right\}^{a}
$$


where $F_{1}$ is the extensional force, $M_{1}, M_{2}$, and $M_{3}$, are the moments about the local $x, y$, and $z$ axes. The total internal forces and moments are based on the $4 \times 4$ stiffness constitutive relation,

$$
\left\{\begin{array}{l}
F_{1} \\
M_{1} \\
M_{2} \\
M_{3}
\end{array}\right\}^{e}=\left[\begin{array}{llll}
K_{11} & K_{12} & K_{13} & K_{14} \\
K_{21} & K_{22} & K_{23} & K_{24} \\
K_{31} & K_{32} & K_{33} & K_{34} \\
K_{41} & K_{42} & K_{43} & K_{44}
\end{array}\right]\left\{\begin{array}{c}
\varepsilon_{x} \\
\kappa_{x} \\
\kappa_{y} \\
\kappa_{z}
\end{array}\right\}
$$

and the piezoelectric forcing is given by

$$
\left\{\begin{array}{l}
F_{1} \\
M_{1} \\
M_{2} \\
M_{3}
\end{array}\right\}^{a}=\left[\begin{array}{llll}
B_{11} & B_{12} & \cdots & B_{1, m} \\
B_{21} & B_{22} & \cdots & B_{2, m} \\
B_{31} & B_{32} & \cdots & B_{3, m} \\
B_{41} & B_{42} & \cdots & B_{4, m}
\end{array}\right]\left\{\begin{array}{c}
v_{1} \\
v_{2} \\
\vdots \\
v_{m}
\end{array}\right\}
$$

where $v_{i}$ is the voltage applied to the ith active ply in the cross section. Each active region along the span has $m$ voltage degrees of freedom, where $m$ is the number of active plies in the cross section.

Since the external forces in the span-wise direction are small, the internal extensional elastic and piezoelectric force are assumed to be in balance. Then by setting $F_{1}$ equal to zero, the first equation can be condensed out by a suitable matrix manipulation.

$$
\begin{gathered}
\left\{\begin{array}{c}
F \\
\hdashline M
\end{array}\right\}=\left\{\begin{array}{c}
0 \\
\hdashline M
\end{array}\right\}=\left[\begin{array}{c:c}
K_{a a} & K_{a b} \\
\hdashline K_{b a} & K_{b b}
\end{array}\right]\left\{\begin{array}{c}
\varepsilon_{x} \\
\hdashline \kappa
\end{array}\right\}-\left[\begin{array}{c}
B_{a} \\
B_{b}
\end{array}\right]\{v\} \\
\mathcal{E}_{x}=\left[K_{a a}\right]^{-1}\left(\left[B_{a}\right]\{v\}-\left[K_{a b}\right]\{\kappa\}\right) \\
{[K]=\left[K_{b b}\right]-\left[K_{b a}\right]\left[K_{a a}\right]^{-1}\left[K_{a b}\right]} \\
{[B]=\left[B_{b}\right]-\left[K_{b a}\right]\left[K_{a a}\right]^{-1}\left[B_{a}\right]} \\
\{M\}=[B]\{v\}-[K]\{\kappa\}
\end{gathered}
$$

This allows for the extensional strains to be eliminated from the wing dynamics while retaining the effects of extension/twist and extension/bend couplings, and the ability to recover extensional stresses and strains for ply failure analysis.

The virtual work done on a wing against the internal elastic and piezoelectric forces by a variation in each of the curvature variables is given by

$$
\begin{aligned}
\delta W^{\text {internal }} & =\int_{s=0}^{L} \delta \vec{\kappa}^{t}\left\{B \vec{v}-K\left(\vec{\kappa}-\vec{\kappa}_{i}\right)\right\} d s \\
& =\delta \underline{\kappa}^{t}\left\{B_{v} \underline{v}-K_{\kappa}\left(\underline{\kappa}-\underline{\kappa}_{i}\right)\right\}
\end{aligned}
$$

\section{Equations of Motion}

After gathering terms from the previous section, the total virtual work done on the vehicle becomes

$$
\begin{aligned}
& \delta W=\delta \underline{q}^{T}\left(-M_{q} \underline{\ddot{q}}-C_{q} \underline{\dot{q}}-K_{q} \underline{q}+B_{1} \underline{v}\right. \\
& \left.+B_{2} \underline{F}^{\text {aero }}+B_{3} \underline{M}^{\text {aero }}+B_{4} \underline{G}_{b}+B_{5} \underline{\kappa}_{i}\right)
\end{aligned}
$$

where all the coefficient matrices except for the stiffness and voltage influence matrices depend on the current wing position.

The structure equations of motion are obtained by requiring that the net virtual work due to each virtual displacement is zero,

$$
\frac{\partial \delta W}{\partial \delta q_{i}}=0
$$

from which the second order equations of motion arise,

$$
\begin{aligned}
& M_{q} \underline{\ddot{q}}+C_{q} \underline{\dot{q}}+K_{q} \underline{q}= \\
& B_{1} \underline{v}+B_{2} \underline{F}^{\text {aero }}+B_{3} \underline{M}^{\text {aero }}+B_{4} \underline{G}_{b}+B_{5} \underline{\kappa}_{i}
\end{aligned}
$$

The equations of motion for the single wing fixed at the root are obtained by retaining only the curvature degrees of freedom for that wing and has the form

$$
\begin{aligned}
& M_{R R} \underline{\ddot{\kappa}}+C_{R R} \underline{\dot{\kappa}}+K_{R R} \underline{\kappa}= \\
& B_{1 R} \underline{v}_{R}+B_{2 R} \underline{F}_{R}^{\text {aero }}+B_{3 R} \underline{M}_{R}^{\text {aero }}+B_{4 R} \underline{G}_{b}+K_{R R} \underline{\kappa}_{i}
\end{aligned}
$$

Aeroelastic Equations of Motion

As described above, the aerodynamic forces and moments can be written in terms of the vehicle motion variables, the induced flow, and gust velocity. The mass, stiffness, and damping matrices are modified to include the corresponding unsteady aerodynamic terms, yielding the aeroelastic equations of motion,

$$
\begin{gathered}
M \underline{\grave{q}} \underline{\ddot{q}}+\dot{\mathfrak{C}}_{\dot{q}} \underline{\dot{q}}+\mathrm{I}^{\grave{q}}+D \underline{\lambda}=\underline{F} \\
\underline{\dot{\lambda}}=F_{1} \underline{\ddot{q}}+F_{2} \underline{\dot{q}}+F_{3} \underline{\lambda} \\
\underline{F}=C_{q} \underline{\dot{q}}_{0}+K_{q} \underline{q}_{0}+D \underline{\lambda}_{0}+B_{1} \underline{v}+B_{2} \underline{F}_{0}^{\text {aero }} \\
+B_{3} \underline{M}_{0}^{\text {aero }}+B_{4} \underline{G}_{b}+B_{5} \underline{\kappa}_{i}+B_{6} \underline{w}
\end{gathered}
$$


where

$$
\begin{gathered}
M \grave{q}=M_{q}-B_{2} F_{\ddot{q}}-B_{3} M_{\ddot{q}} \\
\grave{\mathfrak{C}}=C_{q}-B_{2} F_{\dot{q}}-B_{3} M_{\dot{q}} \\
\grave{\grave{\lambda}}=K-B_{2} F_{q}-B_{3} M_{q} \\
D=-B_{2} F_{\lambda}-B_{3} M_{\lambda} \\
B_{5}=B_{2} F_{w}+B_{3} M_{w}
\end{gathered}
$$

and the single wing aeroelastic model is similarly derived.

\section{State Space Representation}

By adopting a state space format, the structure states can be augmented with the inflow states. Then Eq. (67) becomes

$$
\left[\begin{array}{ccc}
I & 0 & 0 \\
0 & M \grave{q} & 0 \\
0 & -F_{1} & I
\end{array}\right]\left[\begin{array}{c}
\dot{q} \\
\ddot{q} \\
\dot{\lambda}
\end{array}\right]=\left[\begin{array}{ccc}
0 & I & 0 \\
-K_{\grave{a}} & -\grave{\mathfrak{a}} & -D \\
0 & F_{2} & F_{3}
\end{array}\right]\left[\begin{array}{c}
q \\
\dot{q} \\
\lambda
\end{array}\right]+\left[\begin{array}{l}
0 \\
I \\
0
\end{array}\right] \underline{F}
$$

Using the full state vector in Eq. (69), instead of perturbation of the state vector about the instantaneous linearization facilitates a nonlinear time stepping solution effectively since the perturbation equations do not have to be constructed on each time step.

The state space system can be represented in the form

$$
\begin{gathered}
\dot{x}=A(x) x+B(x) u+G(x) w+F(x) \\
y=C(x) x+D u
\end{gathered}
$$

where $u$ is the control input, $y$ is a vector of outputs, which may correspond to strain gages, accelerometers, and rate gyros, $w$ is the gust loading, and $F$ contains all other external loads and disturbance. The state vector is given by

$$
x^{T}=\left\{\begin{array}{llllllll}
\kappa_{R}^{T} & \boldsymbol{\kappa}_{L}^{T} & \phi & \dot{\kappa}_{R}^{T} & \dot{\kappa}_{L}^{T} & \dot{\phi} & \lambda_{R}^{T} & \lambda_{L}^{T}
\end{array}\right\}^{T}
$$

for the roll model and

$$
x^{T}=\left\{\begin{array}{lllll}
\boldsymbol{\kappa}^{T} & \phi & \dot{\kappa}^{T} & \dot{\phi} & \lambda^{T}
\end{array}\right\}^{T}
$$

for the single wing model.

\section{Steady State Deformation}

The steady deformed shape of the wing under the influence of aerodynamic loads, gravity, and applied electric field is given by the nonlinear equation

$$
K\left(\underline{\kappa}-\underline{\kappa}_{i}\right)=B_{1} \underline{v}+B_{2} \underline{F}^{\text {aero }}+B_{3} \underline{M}^{\text {aero }}+B_{4} \underline{G}
$$

which must be solved in an iterative manner since the right hand side matrices and static aerodynamic loads vectors depend on the current deformed geometry. This can be done by successively applying the load vector based on the current geometry and allowing the solution to converge to a final value. Numerical damping may be added for numerical stability. For a stiff wing, the solution is usually obtained within 5 to 7 iterations. As the wings become more flexible, the number of iterations generally increases because of the greater changes in $B_{2}, B_{3}$, and $B_{4}$ as the structure deforms.

\section{Flutter Analysis}

Stability analysis can be conducted by bringing the wing to its steady state nonlinear deflection at various flight conditions and computing the eigenvalues of the linearized system. At the critical flutter speed the real part of the eigenvalues corresponding to the unstable aeroelastic mode become positive, indicating that the mode has negative damping. While the linearized analysis is capable of determining the point of instability, a nonlinear time stepping analysis is needed to identify the presence of a limit cycle if one exists.

\section{Damping}

Internal damping may be added to the model to approximate the true behavior of the wings and to reduce the effects of high frequency structural modes, which may not be well modeled. This can be done in many ways. In the present formulation, the internal damping forces are assumed to enter the structure equations in a similar way as internal elastic forces, i.e., a constitutive equation relating internal forces to strain rates. This enters the discrete model as stiffness proportional damping,

$$
C=\alpha K
$$

where $\alpha$ is solved for which results in the desired first mode damping. Typically, the first mode damping is set to a very small non-zero value $(<.001)$. This has the effect of damping out high frequency structural modes, which do not play an important role in the aeroelastic behavior of the wing.

\section{Numerical Results}

A numerical test model was representing a high-altitude long-endurance vehicle. A graphical representation is shown in Figure 5. These aircraft are designed to fly above $50,000 \mathrm{ft}$ and have half-aspect ratio of around 10:1. In the present study, each wing is $30 \mathrm{~m}$ in length, as shown in Figure 6, and has a single spar running the entire length. The airfoil profile is a NACA 0014 (Figure 7). The active/passive composite layup is 
constant along the span, and is summarized in the appendix. The spanwise stiffness and actuation distributions are shown in Figures 8 and 9, respectively.

A total of four anisotropic piezoelectric actuator (APA) layers are embedded in the airfoil skins; two layers on top and to on bottom, and are arranged to produce both twisting and bending deformation. Two more APA layers are incorporated in the spar.

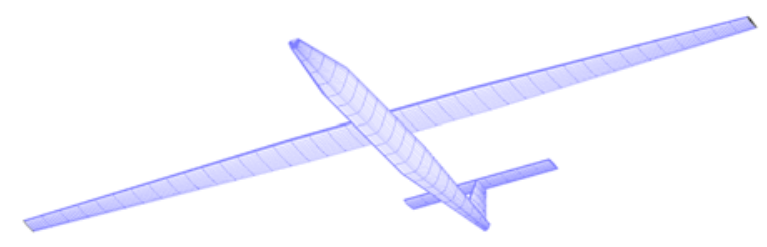

Figure 5: HALE Test Vehicle

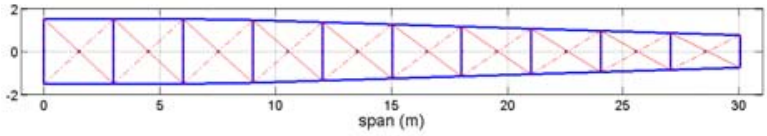

Figure 6: Planform geometry

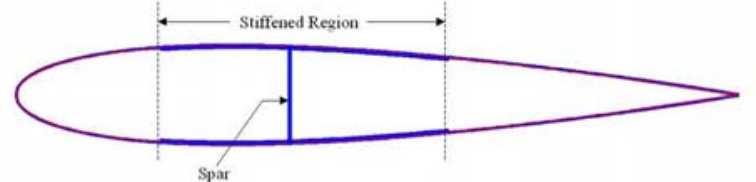

Figure 7: Cross section gometry (NACA0014 airfoil)
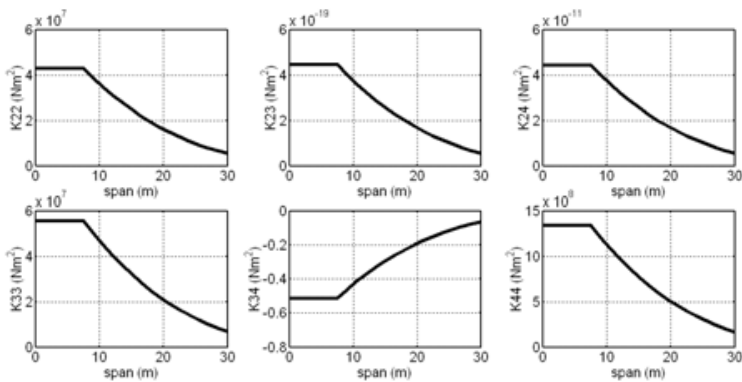

Figure 8: Spanwise Stiffness Distribution
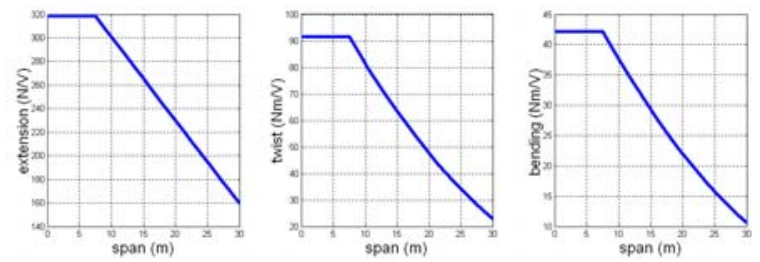

Figure 9: Spanwise actuation distribution

Flight Plan

A simple flight plan was constructed based on the desired altitude profile (Figure 10). The wings are assumed to carry $40 \%$ of the total fuel, distributed over the inboard $60 \%$ of each wing. The fuel mass is assumed to be depleted linearly from the beginning to the end of the flight. A cruise speed of $195 \mathrm{~m} / \mathrm{s}$ and cruise altitude of $19,800 \mathrm{~m}$ are selected. The flight speed profile is constructed such that the dynamic pressure is constant for the duration of the flight. The body trim angle of attack is than solved for at each point along the flight plan (based on the linearized model). Certain points along the flight profile are selected for analysis.
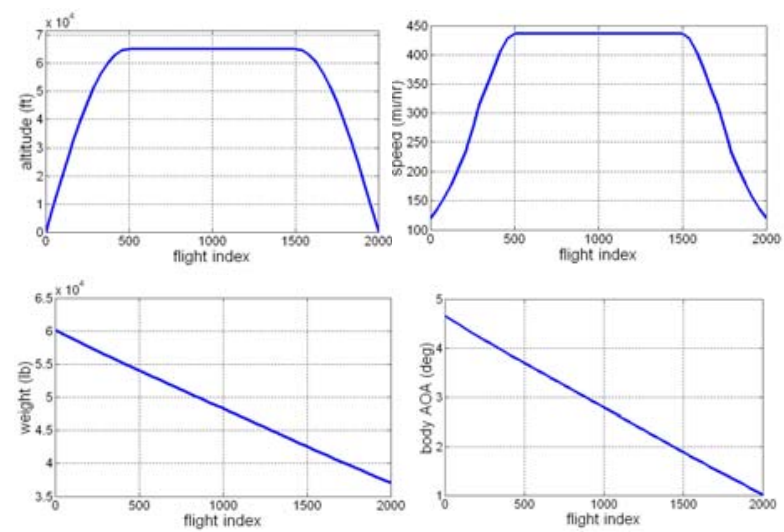

Figure 10: Flight profile definition

Twist Actuation vs. Skin Active Ply Angle

Twist is achieved by adjusting the polarity of the electric field applied to the four active layers on the airfoil surface. Figure 11 shows the twist response of the wing as the active ply angle is varied from 0 to 90 deg. Normally, the maximum twist response would occur at 45 degrees with respect to the spanwise direction. However, since there is coupling with the $+/$ 45-degree spar plies, the maximum twist response occurs when the active plies are oriented at approximately 39 degrees.

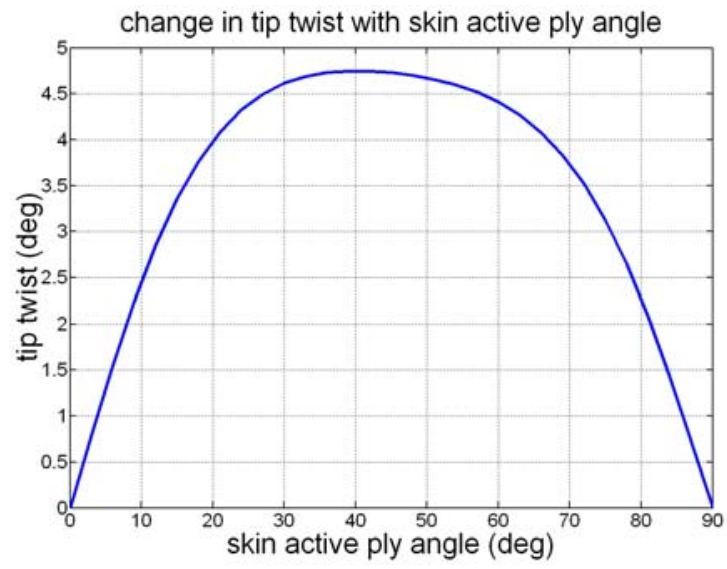

Figure 11: Twist response of the active wing with variation in the orientation of the APA layer angle 


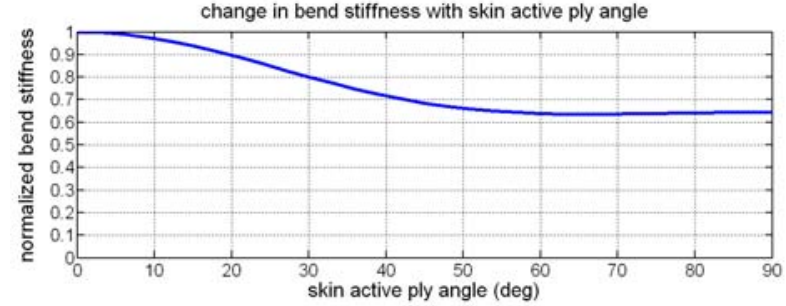

Figure 12: Change in bend stiffness with actuator angle

As seen in Figure 12, changing the fiber direction of the active plies also affects the bending stiffness. The maximum bending stiffness occurs when the active plies are oriented at 0 degrees with respect to the span direction. Rotating the active ply angle by $39 \mathrm{deg}$. corresponds to a $28 \%$ reduction in the bending stiffness.

\section{Stress Distribution in the Deformed Wing}

The steady state solution was obtained for the vehicle at a point in the middle of the flight plan as given in Table 1. The deformed vehicle is shown in Figure 13. Based on the deformed shape of the wing, the stresses on the outer surface were evaluated. Figure 14 shows the spanwise lift distribution and bending moment. Ply stresses were evaluated at several spanwise stations in various plies and are exemplified in Figures 14 and 15.

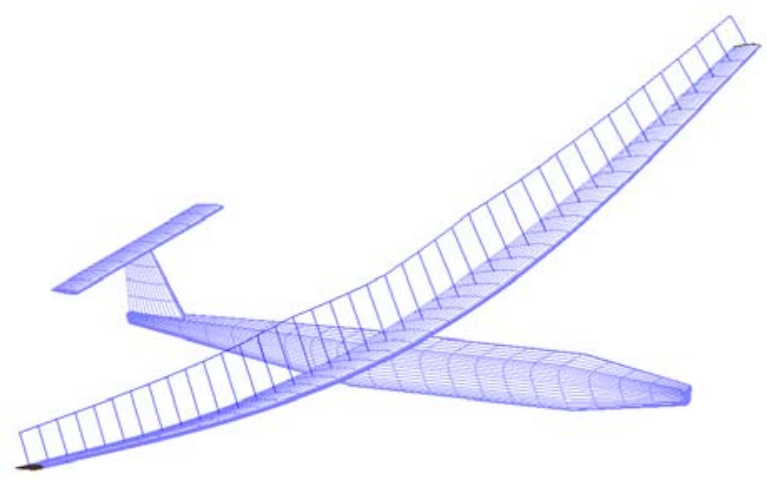

Figure 13: Vehicle at steady state

Table 1: Hale flight data

\begin{tabular}{|l|l|}
\hline Cruise flight speed & $195 \mathrm{~m} / \mathrm{s}$ \\
\hline Cruise altitude & $19,800 \mathrm{~m}$ \\
\hline body trim angle & $2.8 \mathrm{deg}$ \\
\hline Max. fuel mass & $5250 \mathrm{~kg}$ \\
\hline Root attachment angle & $5 \mathrm{deg}$ \\
\hline
\end{tabular}

In Figure 17, the maximum stress in selected plies are plotted as a function of span. As expected, the highest longitudinal stresses occur in the stiffener plies, whith its fibers oriented in the spanwise direction. The 30 deg. graphite composite plies are also highly stressed. The maximum transverse stresses occur in the 4th ply, which is oriented at $39 \mathrm{deg}$.
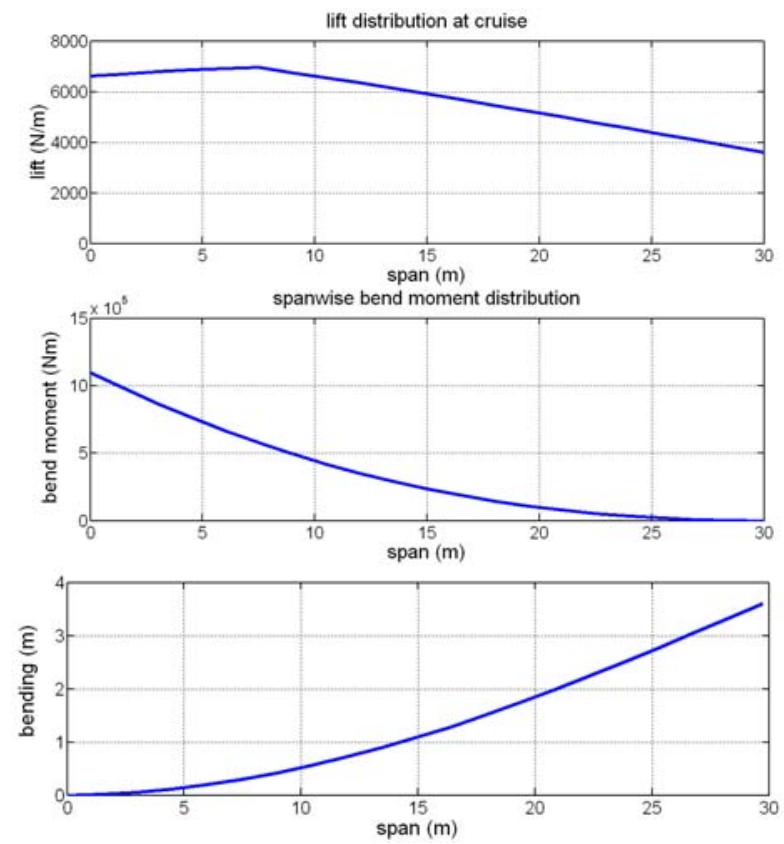

Figure 14: Lift, bending moment, and bending deflection distributions along the wing span

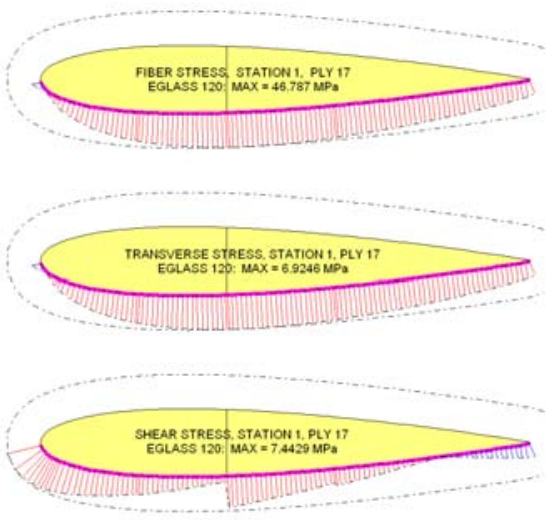

Figure 15: Stress distribution in ply 17 of station 1

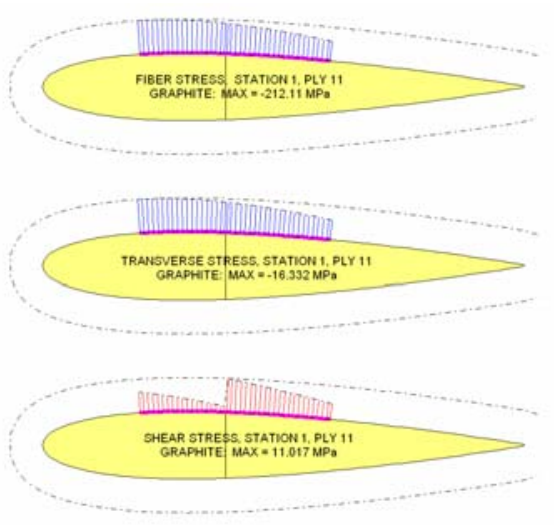

Figure 16: Stress distribution in ply 1 of station 10 


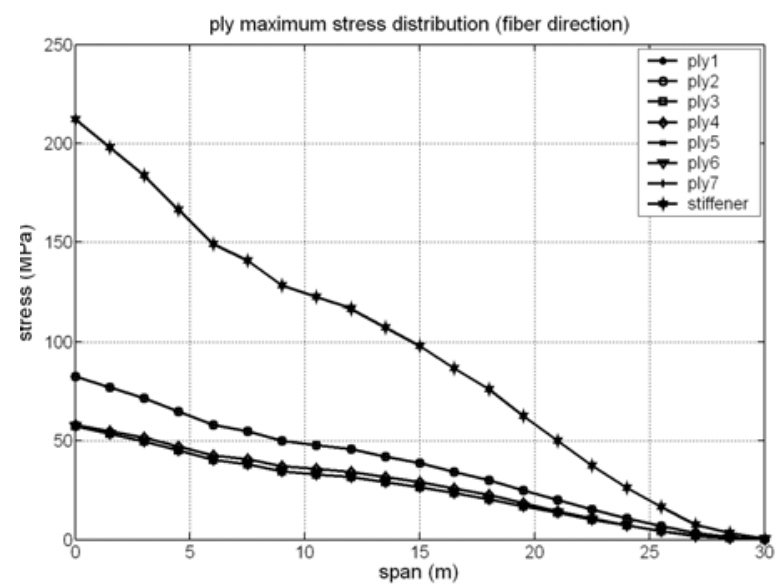

Figure 17: Maximum ply stresses vs. span

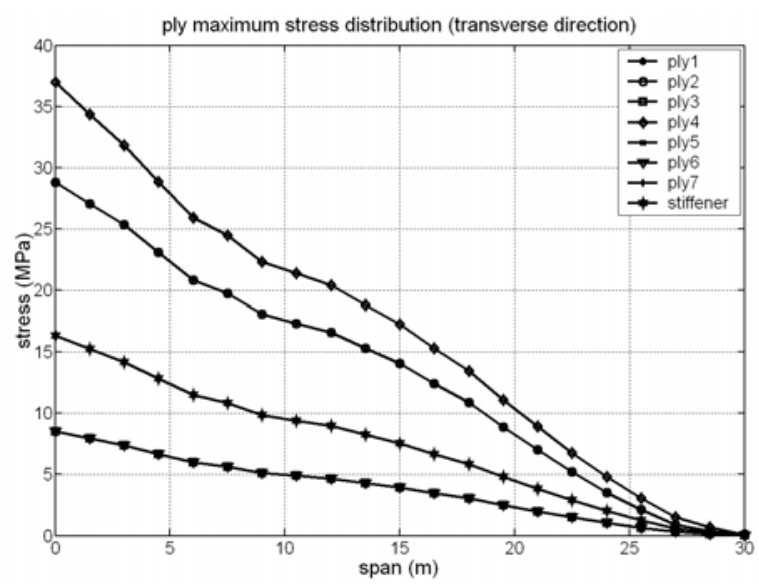

Figure 18: Maximum transverse ply stresses vs. span

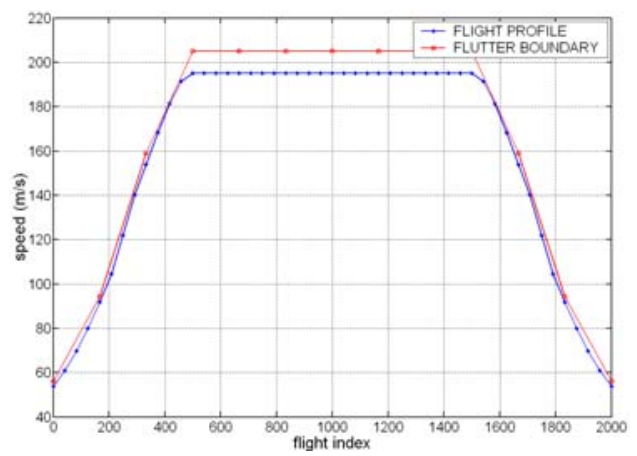

Figure 19: Flutter boundary vs. flight speed profile

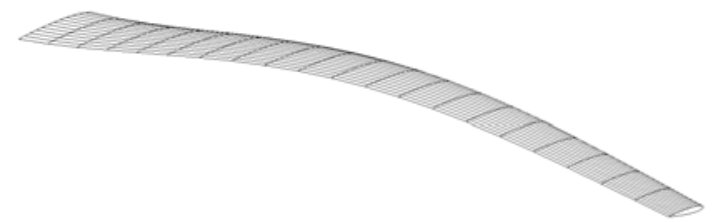

Figure 20: Unstable aeroelastic mode shape
Aeroelastic Stability

The flutter boundary was obtained at several points along the flight profile. At each point, a nonlinear equilibrium solution was obtained and the wing was linearized about that to evaluate the flutter speed. The result is plotted along with the design speed profile in Figure 19. The result shows that the vehicle has approximately $7 \%$ flutter margin in the cruise segment of the flight and it is at the flutter boundary for the other points. This leads either to the need for strucutral redesign, or the continuous use of active controls (not treated here). Figure 20 shows the flutter mode at middle of the cruise segment.

\section{Roll Rate Response}

A comparison between twist actuation and conventional aileron actuation for producing roll is exemplified here. Figure 21 shows the maximum achievable roll rate over the flight plan, based on static calculations. Because the flight speed is lower at the beginning and end of the mission, the roll rate is correspondingly slower. These results show that for this wing, the warping control for roll can provide about $60 \%$ more authority than the aileron control. Figures 22 and 23 show the roll rate time response at three characteristic points along the flight plan for the wing warping and aileron control inputs, respectively. The response to $20 \mathrm{deg}$. flap deflection is shown for comparison. As would be expected, the response in the landing phase is the fastest, since the fuel in the wings has been used up, reducing the roll inertia. The maximum roll rate at takeoff and landing are about the same since the flight speed and altitude are the same.
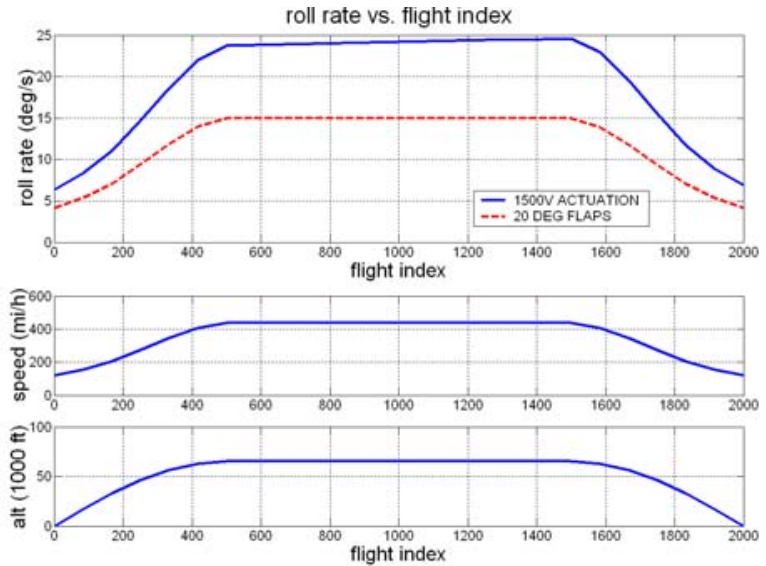

Figure 21: Maximum roll rate vs. flight index 


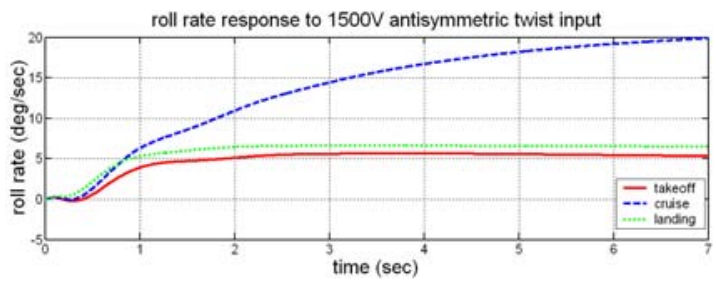

Figure 22: Roll rate response due to wing warping control input at different flight conditions

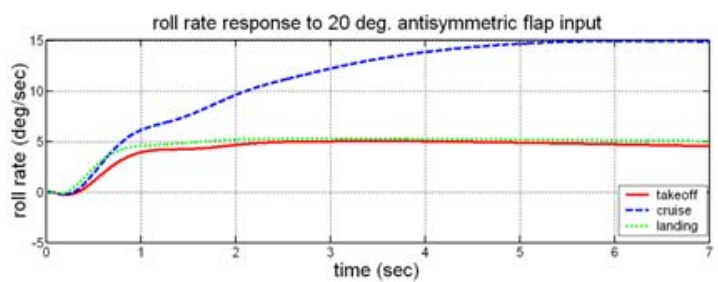

Figure 23: Roll rate response due to 20-deg. aileron control input at different flight conditions

Due to the wing flexibility, the application of sudden aileron or actuator input tends to couple into the first anti-symmetric roll mode of the vehicle, shown in figure 24, delaying the roll angle response slightly.

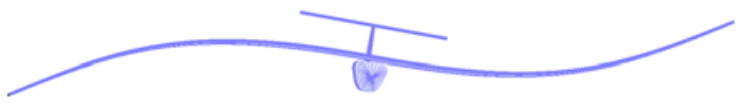

Figure 24: First antisymmetric roll mode $(2.21 \mathrm{~Hz})$

\section{Conclusions}

This paper presented a method for modeling the effects of internal anisotropic strain actuation on the behavior of highly flexible high-aspect ratio wings. The numerical studied presented in this paper showed some of the capabilities of the active aeroelastic formulation in dealing with a HALE-class aircraft. For the proposed design, twisting the wing with embedded anisotropic piezocomposite actuators provides an improvement in performance when compared with traditional ailerons and shows the potential for primary wing control without discrete control surfaces. A nonlinear beam model was developed in order to represent large (coupled) bending and twisting deformations. By dealing with strain variables instead of displacements, the formulations allows for the exact solution of the elastica problem in a very efficient manner. To study the effectiveness of active wings to generate aircraft roll control, a two-wing formulation with freedom to roll was developed based on the strain-based beam formulation. Both wing warping and aileron roll controls are included in the present study. The resulting framework is capable of representing the large deformations associated with the flexible wing with the aeroelastic equations in state-space form. The low-order resulting model allows for control synthesis and simulation. The present implementation provides the capability for integrated structure and control preliminary design for active flexible wings and the exploration of active aeroelastic tailoring concepts.

\section{Acknowledgements}

The second author gratefully acknowledges C.S. Draper Laboratory for providing his fellowship for graduate studies. This work is sponsored by AFOSR under grant F49620-01-1-0133 and by AFOSR-DARPAís Single Crystal Program through Boeing contract No. KH2679. The AFOSR technical monitors are Drs. Daniel Segalman and Dean Mook. The AFOSR-DARPA program managers are Drs. Spencer $\mathrm{Wu}$ and Wallace Smith.

\section{Appendix}

The properties of the HALE wing model used in example presented in this paper is summarized below.

\begin{tabular}{|c|c|c|c|c|}
\hline \multicolumn{5}{|c|}{ WING } \\
\hline \multicolumn{2}{|l|}{ Length } & \multicolumn{3}{|c|}{\begin{tabular}{l|l}
$30 \mathrm{~m}$ \\
\end{tabular}} \\
\hline \multicolumn{2}{|c|}{ Length to start tapering } & \multicolumn{3}{|c|}{7.5} \\
\hline \multicolumn{2}{|c|}{ Root chord } & \multicolumn{3}{|c|}{3} \\
\hline \multicolumn{2}{|l|}{ Tip chord } & \multicolumn{3}{|c|}{1.5} \\
\hline \multicolumn{2}{|l|}{ Airfoil } & \multicolumn{3}{|c|}{ NACA 0014} \\
\hline \multicolumn{5}{|c|}{ Top and Bottom Surfaces } \\
\hline Material & $\begin{array}{l}\text { Thick } \\
(\mathrm{mm})\end{array}$ & $\begin{array}{l}\text { Angle } \\
\text { (deg) }\end{array}$ & $\begin{array}{l}\text { Start } \\
\text { (chrds) }\end{array}$ & $\begin{array}{l}\text { Stop } \\
\text { (chrds) }\end{array}$ \\
\hline E-glass & 1.143 & $0 / 90$ & 0 & 1 \\
\hline APA & 4.360 & -39 & 0 & 1 \\
\hline E-glass & 1.143 & $0 / 90$ & 0 & 1 \\
\hline APA & 4.360 & 39 & 0 & 1 \\
\hline E-glass & 4.932 & $0 / 90$ & 0 & 1 \\
\hline APA & 0 & -39 & 0 & 1 \\
\hline Graphite & 2.286 & 0 & 0.2 & 0.6 \\
\hline \multicolumn{5}{|c|}{ Spar } \\
\hline Location & \multicolumn{4}{|c|}{$38 \%$ from LE } \\
\hline Material & \multicolumn{2}{|c|}{ Thick (mm) } & \multicolumn{2}{|c|}{ Angle (deg) } \\
\hline APA & \multicolumn{2}{|c|}{2.180} & \multicolumn{2}{|c|}{45} \\
\hline Graphite & \multicolumn{2}{|l|}{2.286} & \multicolumn{2}{|l|}{-45} \\
\hline Graphite & \multicolumn{2}{|l|}{2.286} & \multicolumn{2}{|l|}{45} \\
\hline APA & \multicolumn{2}{|l|}{2.180} & \multicolumn{2}{|l|}{-45} \\
\hline
\end{tabular}

The material properties of the composite layers used in HALE wing model are summarized below.

\begin{tabular}{|l|c|c|c|}
\hline & E-glass & Graphite & APA \\
\hline Mass $\left(\mathrm{kg} / \mathrm{m}^{3}\right)$ & 1700 & 1500 & 4100 \\
\hline $\mathrm{Q}_{11}(\mathrm{GPa})$ & 20 & 73 & 34 \\
\hline $\mathrm{Q}_{12}(\mathrm{GPa})$ & 2.9 & 5.6 & 7.5 \\
\hline $\mathrm{Q}_{22}(\mathrm{GPa})$ & 20 & 73 & 17 \\
\hline $\mathrm{Q}_{33}(\mathrm{GPa})$ & 4.1 & 6.6 & 5.1 \\
\hline Free strain $(\mu \varepsilon)$ & - & - & 2700 \\
\hline
\end{tabular}




\section{References}

[1] Lin, C. Y. and Crawley, E. F., "Design Considerations for a Strain Actuated Adaptive Wing for Aeroelastic Control," Journal of Intelligent Material Systems and Structures, Vol. 6 - May 1995

[2] McGowan, A. R., Heeg, J., and Lake, R., ì Results of Wind-Tunnel Testing from the Piezoelectric Aeroelastic Tailoring Investigation, $\hat{1}$ In Proceedings of the 37th AIAA/ASCE/AHS/ASC Structures, Structural Dynamics and Materials Conference, Salt Lake City, Utah, April 1996.

[3] Librescu, L. and Na, S., ì Vibration and Dynamic Response Control of Elastically Tailored Nonuniform Adaptive Aircraft Wingsî, In Proceedings of the 41st AIAA/ASME/ASCE/ AHS/ASC Structures, Structural Dynamics, and Material Conference and Exhibit, Atlanta, GA, April 3-6, 2000, AIAA-2000-1628.

[4] Pendleton, E. W., Griffin, K. E., Kehoe, M., and Perry, B., ì A Flight Research Program for the Active Aeroelastic Wing Concept,î In Proceedings of the 37th AIAA/ASCE/AHS/ASC Structures, Structural Dynamics and Materials Conference, Salt Lake City, Utah, April 1996.

[5] Khot, N. S. and Zweber, J. V., "Lift Efficient Composite Flexible Wing for Rolling Maneuver Without Ailerons," In Proceedings of the 41st AIAA/ASME/ASCE/ AHS/ASC Structures, Structural Dynamics, and Material Conference and Exhibit, Atlanta, GA, April 3-6, 2000, AIAA-2000-1333.

[6] Jones, R. I., "The Design Challenge of High Altitude Long Endurance (Hale) Unmanned Aircraft," The Aeronautical Journal, June, 1999, pp. 273-280.

[7] Patil, M. J., Hodges, D. H., and Cesnik, C. E. S., ì Nonlinear Aeroelasticity and Flight Dynamics of High-Altitude Long-Endurance Aircraft,î J. of Aircraft, Vol. 38, No. 1, January-February 2001, pp. 95ñ103.

[8] Drela, M., "Integrated Simulation Model For Preliminary Aerodynamic, Structural, and Control-Law Design of Aircraft", AIAA-99-1394.

[9] Ortega-Morales, M. and Cesnik, C. E. S., "Modeling and Control of the Aeroelastic Response of Highly Flexible Active Wings," AMSL Report \#2000-2, 2000.

[10] Cesnik, C. E. S. and Ortega-Morales, M., ì Active Aeroelastic Tailoring of Slender Flexible Wings,î
International Forum on Aeroelasticity and Structural Dynamics, Madrid, Spain, June 5ó 7, 2001

[11] Peters, D. A. and Johnson, M. J., "Finite-State Airloads for Deformable Airfoils on Fixed and Rotating Wings", In Symposium on Aeroelasticity and Fluid/Structure Interaction, Proceedings of the Winter Annual Meeting. ASME, November 6 -11, 1994.

[12] Peters, D. A., Karunamoorthy, S., and Cao, W. M., "Finite State Induced Flow Models; Part I: TwoDimensional Thin Airfoil", Journal of Aircraft, Vol. 32, No. 2, March ñ April 1995, pp. 313-322.

[13] Cesnik, C. E. S. and Shin, S.-J., ì On the Modeling of Active Helicopter Blades,î Int. Journal of Solids and Structures, Vol. 38, Nos. 10-13, March 2001, pp. 17651789.

[14] Cesnik, C. E. S. and Ortega-Morales, M., "Active Beam Cross-Sectional Modeling" J. of Intelligent Material Systems \& Structures, 2002 (to appear). 